\title{
Change in the manifestations of asthma and asthma-related traits in childhood: a latent transition analysis
}

\author{
Frances L. Garden ${ }^{1,2,3,4}$, Judy M. Simpson ${ }^{1}$, Craig M. Mellis ${ }^{5}$ and \\ Guy B. Marks ${ }^{2,3}$ for the CAPS Investigators
}

Affiliations: ${ }^{1}$ Sydney School of Public Health, University of Sydney, Sydney, Australia. ${ }^{2}$ South Western Sydney Clinical School, University of New South Wales, Sydney, Australia. ${ }^{3}$ Woolcock Institute of Medical Research, Sydney, Australia. ${ }^{4}$ Ingham Institute of Applied Medical Research, Sydney, Australia. ${ }^{5}$ Central Clinical School, University of Sydney, Sydney, Australia.

Correspondence: Frances L. Garden, Sydney School of Public Health, Edward Ford Building A27, The University of Sydney, NSW, 2006, Australia. E-mail: frances.gardendasydney.edu.au

ABSTRACT It is known that asthma is a heterogeneous entity whose manifestations vary with age. Our objective was to examine changes in the manifestation of asthma and asthma-related traits in childhood by defining empirically derived childhood asthma phenotypes and examining their transitions over time.

To define the phenotypes we used data on respiratory symptoms, healthcare utilisation, medications, spirometry, airway hyperresponsiveness (AHR), exhaled nitric oxide concentration and atopy from a birth cohort recruited on the basis of having a first-degree relative with asthma. Data were acquired at ages $1.5-11.5$ years and analysed using latent transition analysis.

In a study population of 370 participants, we classified subjects into four phenotypes: 1) nonatopic, few symptoms (prevalence range from 1.5 to 5 years: 52-60\%), 2) atopic, few symptoms (3-21\%), 3) nonatopic, asthma and rhinitis symptoms (13-35\%), and 4) atopic, asthma and rhinitis symptoms (2-14\%) in early childhood; and 1) nonatopic, no respiratory disease (prevalence range from 8 to 11.5 years: 41-46\%), 2) atopic, no respiratory disease $(23-33 \%), 3)$ nonatopic, asthma symptoms, no AHR or airway inflammation (8-12\%) and 4) atopic asthma (19\%) in mid-childhood. Transitioning between phenotypes was common in early childhood, but less common in later childhood.

This analysis represents the first attempt to incorporate longitudinal patterns of several manifestations of asthma into a single model to simultaneously define phenotypes and examine their transitions over time. It provides quantitative support for the view that asthma is a heterogeneous entity, and that some children with wheeze and other respiratory symptoms in early life progress to asthma in mid-childhood, while others become asymptomatic.

@ERSpublications

New objective childhood asthma phenotypes show transitioning between phenotypes is common in early not late childhood http://ow.ly/RT5qz

For editorial comment see Eur Respir J 2016; 47: 362-365 [DOI: 10.1183/13993003.02011-2015].

This article has supplementary material available from erj.ersjournals.com

Received: Feb 172015 | Accepted after revision: Aug 172015 | First published online: Oct 222015

Conflict of interest: Disclosures can be found alongside the online version of this article at erj.ersjournals.com

Support statement: This study was supported by the University of Sydney and the National Health and Medical Research Council of Australia. Funding information for this article has been deposited with FundRef.

Copyright @ERS 2016 


\section{Introduction}

Heterogeneity in the manifestations of asthma and related respiratory diseases in childhood is likely to have important implications for the investigation of disease aetiology, mechanisms and management, as well as for population monitoring. So far, attempts to explore this heterogeneity and define distinct phenotypes of asthma in children have focused on either the time course of wheeze [1-4] and other symptoms [5-7] during childhood or on cross-sectional classification of disease manifestations at specified ages [8-12]. Recently, the use of unsupervised, data-driven statistical approaches, such as cluster analysis $[9,11,12]$ and latent class analysis $[2,3,8,10,13]$, has emerged as complementary to approaches based on the application of a priori definitions $[1,14]$ to aid in identifying and defining objective, novel or previously unrecognised phenotypes. Phenotypes previously identified have varied due to differences in the populations and attributes included in the models. However, the application of data-driven approaches has yielded phenotypic classifications that are clinically meaningful and interpretable $[12,15]$, and that are relevant to prognosis [6].

Despite these advances, the application of data-driven approaches to define longitudinal phenotypes to the multiple manifestations of asthma and related disorders during childhood remains largely unexplored. This is important because these manifestations are not independent. Indeed, the various manifestations of asthma, such as cough, wheeze, atopy, impaired lung function, airway hyperresponsiveness (AHR) and airway inflammation, are known to be associated with each other and to vary with age [16]. Two studies have examined transitions between phenotypes in preschool children that were defined based on wheeze alone [17] or with multiple clinical features including wheeze triggers [18]. Data from the Isle of Wight study have been used to describe transitions during childhood in sex-specific phenotypes that were defined based on two measures: physician-diagnosed asthma and wheeze [19]. In adults, latent transition analysis (LTA) has been used to define asthma phenotypes longitudinally [20]. However, to the best of our knowledge, there has been no previous attempt to incorporate the longitudinal pattern of several disease manifestations into one statistical model to simultaneously define phenotypes, and to examine transitions in asthma and related disorders in children.

We propose that, during childhood, asthma consists of several underlying traits with time-varying manifestations. Our primary objective was to examine changes in the manifestation of asthma and asthma-related traits in childhood by quantitatively describing and classifying these underlying traits as defined by phenotypes and examining their transitions over time. A secondary objective was to explore the relationship of various predictive factors to these phenotypes. This was done using observations of several manifestations of asthma and related diseases that were made at various ages during early and mid-childhood in a birth cohort of children at risk of asthma.

\section{Methods}

Additional details are provided in the online supplementary material.

\section{Design and population}

We used data from the first 11.5 years of life in the Childhood Asthma Prevention Study (CAPS) cohort. CAPS began as a randomised controlled trial investigating the effectiveness of a house dust mite (HDM) avoidance and an $\omega-3$ fatty acid supplementation intervention from birth to 5 years for the primary prevention of asthma [21]. Pregnant women, whose unborn children were at increased risk of developing asthma because one or more parents or siblings had current asthma or wheezing, were recruited from antenatal clinics in western and south-western Sydney, Australia, from 1997 to 2000. Babies from multiple births, whose gestational age was $<36$ weeks or birthweight $<2.5 \mathrm{~kg}$, who were hospitalised for $>1$ week or had serious illness, those with a pet cat at home and those whose families were strict vegetarians were excluded [22]. Among 7171 subjects screened for inclusion, 29\% (2095) met the eligibility criteria, of whom $29 \%$ (616) consented to enrolment and randomisation [23]. Further details of the study design, interventions, population, and results of the trial at ages 5, 8 and 11.5 years have been described previously $[21,22,24,25]$. Here, we present a longitudinal analysis of the data. We restricted this analysis to subjects who completed the 11.5-year assessment (370/616).

\section{Measures}

Details of the assessments conducted on this cohort have been described previously [21, 22, 24, 25] and relevant details are summarised here. To define the phenotypes, we used data collected during clinical assessments conducted at ages 1.5, 3, 5, 8 and 11.5 years, including information on respiratory symptoms (wheeze, cough and sneezing or running nose), healthcare utilisation (visited an emergency department or hospital admission for wheeze, asthma, bronchitis or bronchiolitis), treatment (preventer/controller or bronchodilator medication), lung function (percent predicted forced expiratory volume in $1 \mathrm{~s}$ (FEV1)), non-specific AHR, exhaled nitric oxide concentration (eNO) and atopy (inhalant and ingested allergens). 
Data on the following risk factors were obtained and used as predictors of phenotypes: participant's sex, CAPS randomised intervention groups (active/control HDM intervention group and diet intervention group) [22], maternal smoking in pregnancy, exposure to household tobacco smoke before 1.5 years, breastfeeding $\geqslant 6$ months, eczema at 1.5 years [26-28], daycare attendance before 1.5 years, older siblings and parental education attainment.

\section{Statistical analysis}

To define the phenotypes and examine changes over time we used LTA, estimated by the full information maximum likelihood method to handle the presence of missing data, implemented in Mplus version 7 [20, 29, 30]. The model input variables were the measures described above. Percent predicted FEV1, AHR and eNO were only available at ages 8 and 11.5 years.

LTA simultaneously defines classes, which were interpreted as phenotypes, at each assessment time (1.5, 3 , 5,8 and 11.5 years) and models transitions between the classes. We used the model to simultaneously estimate class prevalence and item response probabilities (i.e. proportion of children in each class in whom the binary variable is "positive") at each age and probability of transition between classes at subsequent ages. We used a likelihood ratio test to determine whether the best-fitting model was obtained by allowing class structures to vary at all times (unconstrained model) or between early (1.5-5 years) and mid-childhood (8-11.5 years) (constrained model) [29,31]. To determine the optimal number of classes within the selected level of constraint, we ran a series of models with incrementing class size and examined model fit statistics (Bayesian information criteria (BIC), adjusted BIC, Akaike's information criteria and entropy) and interpretability of the resulting class structure [29]. We examined the item response probabilities to select an appropriate interpretation and label for each class (phenotype).

We tested potential predictors of class membership (phenotype) using multinomial logistic regression implemented in SAS version 9.3 (SAS Institute, Cary, NC, USA). In this analysis individuals were assigned to the class with the highest probability of membership for that individual. We also examined the frequency distribution of other symptom variables, current eczema and questionnaire-defined current asthma within the classes at each time point. Questionnaire-defined current asthma was defined as wheeze in the last 12 months (at that age) and ever been diagnosed with asthma by a doctor (reported at ages 18 months, and 3, 5, 8 and 11.5 years).

\section{Results}

\section{Participant characteristics}

Of the 616 subjects recruited into CAPS at birth, 370 (60\%) who participated in the 11.5-year assessment were included in this analysis. Characteristics of the sample are described in table 1. Compared with those lost to follow-up, those included in this analysis were more likely to have mothers who did not smoke during pregnancy, were older, better educated or in full-time employment and also were more likely to have fathers who were older, better educated or in full-time employment [25].

LTA

A model that was allowed to have a different class structure during early childhood (1.5-5 years) and mid-childhood (8-11.5 years), but was constrained to have the same class structure within these two periods, was chosen as the optimal LTA model (table 2). Four class models had the optimal model fit statistics for both these periods and therefore were selected. The four-class LTA model had a high entropy value (0.91), indicating there was a strong, clear delineation of phenotypes [32]. Further details on the statistical basis for the choice of this model structure are provided in the online supplementary material.

\section{LTA phenotype descriptions}

The structure of the four classes (phenotypes) is shown in table 3 and the prevalence of each phenotype at all ages is shown in figure 1.

During early childhood (1.5-5 years) the phenotypes were labelled: " 1 A: nonatopic, few symptoms, early childhood" (prevalence range: $60 \%$ at 1.5 years to $52 \%$ at 5 years) due to the low probabilities $(<40 \%)$ of each attribute in the model; "1B: atopic, few symptoms, early childhood" (3\% at 1.5 years to $21 \%$ at 5 years) due to the high probability of atopy to inhalant allergens (95\%), moderate probability of atopy to ingested allergens (14\%) and only moderate probability of respiratory symptoms (wheeze 12\%); "1C: nonatopic, asthma and rhinitis symptoms, early childhood" (35\% at 1.5 years to $13 \%$ at 5 years) due to the low probability of atopy $(<6 \%)$ and high probability of symptoms (wheeze $85 \%$ ) and medication use (bronchodilators 91\%); and "1D: atopic, asthma and rhinitis symptoms, early childhood" (2\% at 1.5 years to $14 \%$ at 5 years) due to the high probability of respiratory symptoms (wheeze 92\%), treatment (bronchodilators 97\%) and atopy (inhalant allergens 100\%). The main attributes that differentiated the phenotypes in early childhood were atopy (inhalant allergens 1-100\%) and symptoms (wheeze 12-92\%). 
TABLE 1 Characteristics of the study sample $(n=370)$ and description of the variables used in the latent transition analysis model

\begin{tabular}{ccccc}
\multicolumn{5}{c}{ Age years } \\
\hline 1.5 & 3 & 5 & 8 & 11.5
\end{tabular}

\begin{tabular}{|c|c|c|c|c|c|}
\hline \multicolumn{6}{|l|}{ Symptoms } \\
\hline Cough lasting 1 week or more in the last $18 / 12$ months $^{\#}$ & $182 / 370(49.2)$ & $198 / 370(53.5)$ & $172 / 369(46.6)$ & $135 / 343(39.4)$ & $59 / 369(16.0)$ \\
\hline $\begin{array}{l}\text { Sneezing or running nose lasting } 1 \text { week or more in the } \\
\text { last } 18 / 12 \text { months" }\end{array}$ & $199 / 370(53.8)$ & $171 / 370(46.2)$ & $152 / 369(41.2)$ & & \\
\hline $\begin{array}{l}\text { Sneezing or running nose in the last } 12 \text { months when } \\
\text { he/she did not have a cold or the flu }\end{array}$ & & & & $89 / 341(26.1)$ & $140 / 370(37.8)$ \\
\hline \multicolumn{6}{|l|}{ Healthcare utilisation } \\
\hline $\begin{array}{l}\text { Attended a hospital emergency department or admitted } \\
\text { to hospital for wheeze, bronchitis, bronchiolitis or } \\
\text { asthma in the last } 18 / 12 \text { months }{ }^{\#}\end{array}$ & $52 / 370(14.1)$ & 20/370 (5.4) & 14/369 (3.8) & $7 / 343(2.0)$ & $8 / 370(2.2)$ \\
\hline \multicolumn{6}{|l|}{ Treatment use } \\
\hline $\begin{array}{l}\text { Preventer/controller medication used in the last } \\
12 \text { months }\end{array}$ & 42/370 (11.4) & $55 / 370(14.9)$ & $63 / 369(17.1)$ & $62 / 343(18.1)$ & $65 / 370(17.6)$ \\
\hline Bronchodilator medication used in the last 12 months & $163 / 370(44.0)$ & $172 / 370(46.5)$ & $131 / 369(35.5)$ & $121 / 343(35.2)$ & $118 / 369(32.0)$ \\
\hline \multicolumn{6}{|l|}{ ( } \\
\hline Inhalant atopy & 25/361 (6.9) & $81 / 366(22.1)$ & $127 / 353(36.0)$ & 140/308 (45.5) & $160 / 290(55.2)$ \\
\hline
\end{tabular}

Data are presented as $\mathrm{n} / \mathrm{N}(\%)$. FEV1: forced expiratory volume in $1 \mathrm{~s} ; \mathrm{PD}_{20} \mathrm{FEV}_{1}$ : provocative dose causing a $20 \%$ fall in $\mathrm{FEV} 1 .{ }^{\#}$ : in the previous 18 months was used at the 1.5- and 3-year assessments, and in the previous 12 months was asked at all other assessments.

During mid-childhood (8-11.5 years) the phenotypes were labelled: "2A: nonatopic, no respiratory disease, mid-childhood" ( $46 \%$ at 8 years to $41 \%$ at 11.5 years), "2B: atopic, no respiratory disease, mid-childhood" ( $23 \%$ at 8 years to $33 \%$ at 11.5 years), "2C: nonatopic, asthma symptoms, no AHR or airway inflammation, mid-childhood" ( $12 \%$ at 8 years to $8 \%$ at 11.5 years) and " $2 \mathrm{D}$ : atopic, asthma, mid-childhood" (19\% at both 8 years and 11.5 years). The phenotypes in mid-childhood were very similar to those in early childhood. The additional attributes (FEV1, AHR and eNO) during this period enabled the phenotypes 2C and $2 \mathrm{D}$ to be further refined. The main attributes that differentiated the phenotypes were atopy (inhalant allergens 3-100\%), symptoms (wheeze 4-79\%), AHR (6-54\%) and elevated eNO (4-70\%).

The relative prevalence of the atopic phenotypes $(1 \mathrm{~B}, 2 \mathrm{~B}, 1 \mathrm{D}$ and $2 \mathrm{D})$ increased with age during the study period, while the prevalence of the nonatopic phenotypes (1A, $2 \mathrm{~A}, 1 \mathrm{C}$ and $2 \mathrm{C})$ decreased with age.

The distribution of other characteristics among the phenotypes is shown in online supplementary tables E2 and E3. Participants in the symptomatic phenotypes (1C, 1D, 2C and 2D) had the highest prevalence of the other respiratory symptoms and allergic disease symptoms at all ages.

TABLE 2 Model fit statistics from the constrained and unconstrained latent transition analysis models ( $\mathrm{n}=370$ )

\begin{tabular}{|c|c|c|c|c|c|c|}
\hline Model & Parameters n & $\begin{array}{c}\text { Akaike's } \\
\text { information criteria }\end{array}$ & $\begin{array}{c}\text { Bayesian } \\
\text { information criteria }\end{array}$ & $\begin{array}{l}\text { Adjusted Bayesian } \\
\text { information criteria }\end{array}$ & Entropy & p-value \\
\hline \multicolumn{7}{|l|}{ Unconstrained } \\
\hline Three-class & 164 & 13220 & 13862 & 13342 & 0.905 & \\
\hline Four-class & 235 & 12913 & 13833 & 13087 & 0.907 & \\
\hline \multicolumn{7}{|l|}{ Constrained ๆ } \\
\hline Four-class & 121 & 13072 & 13545 & 13161 & 0.906 & $<0.001$ \\
\hline
\end{tabular}


TABLE 3 Item response probabilities for attributes within each phenotype, by age group, from the constrained latent transition analysis model

\begin{tabular}{|c|c|c|c|c|c|c|c|}
\hline \multicolumn{4}{|c|}{ Phenotype at $1.5,3$ and 5 years } & \multicolumn{4}{|c|}{ Phenotype at 8 and 11.5 years } \\
\hline $1 \mathrm{~A}$ & $1 B$ & $1 \mathrm{C}$ & 1D & $2 A$ & 2B & 2C & 2D \\
\hline 0.352 & 0.412 & 0.802 & 0.699 & 0.126 & 0.113 & 0.599 & 0.537 \\
\hline 0.151 & 0.115 & 0.852 & 0.921 & 0.044 & 0.126 & 0.790 & 0.703 \\
\hline 0.385 & 0.398 & 0.666 & 0.571 & 0.155 & 0.361 & 0.407 & 0.601 \\
\hline 0.005 & 0.000 & 0.244 & 0.187 & 0.000 & 0.000 & 0.041 & 0.089 \\
\hline 0.009 & 0.033 & 0.388 & 0.487 & 0.005 & 0.010 & 0.517 & 0.637 \\
\hline 0.172 & 0.181 & 0.913 & 0.965 & 0.076 & 0.098 & 0.964 & 0.941 \\
\hline 0.039 & 0.139 & 0.054 & 0.266 & 0.016 & 0.079 & 0.000 & 0.215 \\
\hline 0.013 & 0.951 & 0.027 & 1.000 & 0.033 & 1.000 & 0.164 & 0.989 \\
\hline & & & & 0.078 & 0.050 & 0.039 & 0.180 \\
\hline & & & & 0.126 & 0.113 & 0.061 & 0.537 \\
\hline & & & & 0.044 & 0.126 & 0.094 & 0.703 \\
\hline
\end{tabular}
constrained to have one class structure for ages 1.5, 3 and 5 years, and another class structure for ages 8 and 11.5 years. 1A: nonatopic, few symptoms, early childhood; 1B: atopic, few symptoms, early childhood; 1C: nonatopic, asthma and rhinitis symptoms, early childhood; 1D: atopic, asthma and rhinitis symptoms, early childhood; 2A: nonatopic, no respiratory disease, mid-childhood; 2B: atopic, no respiratory disease, mid-childhood; 2C: nonatopic, asthma symptoms, no airway hyperresponsiveness or airway inflammation, mid-childhood; 2D: atopic, asthma, mid-childhood. FEV1: forced expiratory volume in 1 s; PD20FEV1: provocative dose causing a $20 \%$ fall in FEV1.

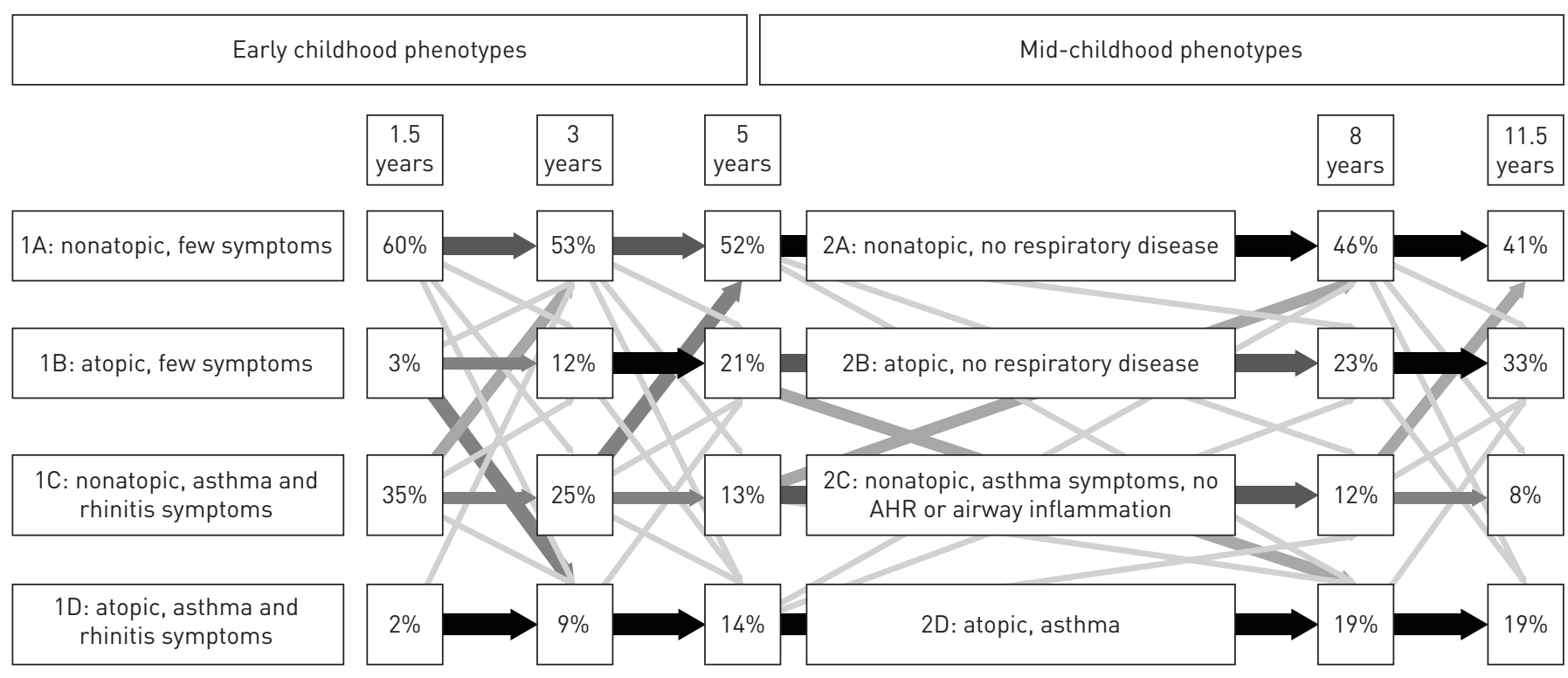

\begin{tabular}{c|c|c|c|cc|}
\multirow{2}{*}{$\begin{array}{c}\text { Transition } \\
\text { probabilities }\end{array}$} & \multicolumn{3}{|c}{$\longrightarrow$} \\
\cline { 2 - 6 } & $1-19$ & $20-39$ & $40-59$ & $60-79$ & $80-100$
\end{tabular}

FIGURE 1 Phenotype prevalence and transition probabilities between the phenotypes at subsequent ages. Prevalence of each phenotype at each age is recorded in the boxes under the age heading. The transition probabilities represent the probability that a member of a given phenotype at a specified age will transition to another given phenotype at the next specified age. Transition probabilities $>0 \%$ are represented by arrows. The width and shading of the arrow represents the transition probability. Phenotype prevalence and transition probabilities are based on the estimated model (exact probabilities are shown in online supplementary table E1). AHR: airway hyperresponsiveness. 
TABLE 4 Association between risk factors or other attributes of the participants and phenotype membership, by age

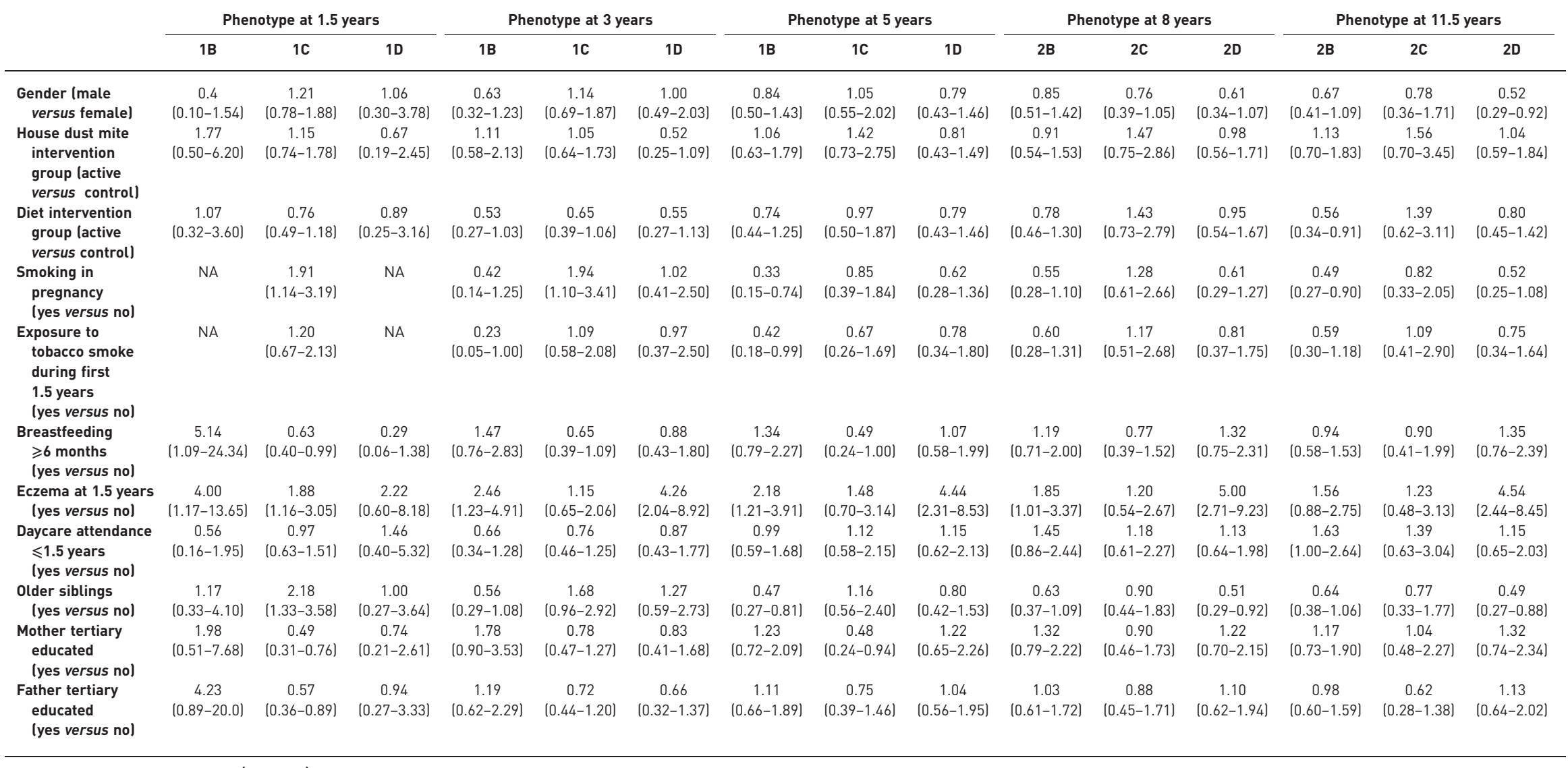

Data are presented as OR (95\% CI) relative to reference groups 1A for early childhood phenotypes and 2A for mid-childhood phenotypes for each age. 1B: atopic, few symptoms, early childhood; 1C: nonatopic, asthma and rhinitis symptoms, early childhood; 1D: atopic, asthma and rhinitis symptoms, early childhood; 2B: atopic, no respiratory disease, mid-childhood;

2C: nonatopic, asthma symptoms, no airway hyperresponsiveness or airway inflammation, mid-childhood; 2D: atopic, asthma, mid-childhood. NA: not available. 


\section{Transitions over time}

Figure 1 shows the transition probabilities of individuals moving from one phenotype at a given age to another phenotype at the subsequent age. During each of the periods (early and mid-childhood) the horizontal arrows represent the probability of transitioning to a phenotype with the same characteristics, while other arrows represent the probabilities of moving to other phenotypes. The transition probabilities between 5 and 8 years reflect the probabilities of moving to phenotypes with similar characteristics.

Transitioning to a different phenotype between ages 1.5 and 3 years was common (average transition probability $12 \%$; range $0-47 \%$ ) (figure 1 ). For all subsequent ages, remaining in the same phenotype classification was common for those in the atopic phenotypes $(1 \mathrm{~B}, 2 \mathrm{~B}, 1 \mathrm{D}$ and $2 \mathrm{D})$ and nonatopic with few symptoms phenotype ( $1 \mathrm{~A}$ and $2 \mathrm{~A}$ ) (average transition probability to another phenotype $4 \%$; range 0 $26 \%$ ) and not common in the nonatopic asthma symptoms phenotype (1C and 2C) who were most likely to transition to the nonatopic, few symptom phenotype (1A and $2 \mathrm{~A}$ ) (average transition probability $34 \%$ ). We compared the transition probabilities in the constrained model with those in the unconstrained models (online supplementary table E5) and found similar results.

We examined the fate at 11.5 years of subjects according to their class at 1.5 years. Of those in the nonatopic, few symptoms phenotype $(1 \mathrm{~A})$ at 1.5 years, $44 \%$ were classified as the similar phenotype (2A) at 11.5 years. Similarly, $55 \%$ in the atopic, few symptoms phenotype (1B) at 1.5 years were classified as the similar phenotype (2B) at 11.5 years. Only $13 \%$ in the nonatopic asthma and rhinitis symptoms phenotype (1C) at 1.5 years were classified as the similar phenotype (2C) at 11.5 years. The majority (70\%) of those in the atopic, asthma and rhinitis symptoms phenotype (1D) were classified as the similar phenotype (2D) at 11.5 years, and were most likely to be classified as this phenotype $(1 \mathrm{D}$ or $2 \mathrm{D})$ at all other ages.

We also identified the 10 most common pathways through all assessments from ages 1.5 to 11.5 years (online supplementary table E6). The most common path was to remain in the nonatopic, few symptoms/ no respiratory disease phenotypes ( $1 \mathrm{~A}$ and $2 \mathrm{~A} ; \mathrm{n}=83,22 \%$ ). Other common paths were to begin in the nonatopic, few symptoms phenotype at ages 1.5 or 3 years, and transition to and remain in the atopic, few symptoms/no respiratory disease phenotype $(1 \mathrm{~B}$ or $2 \mathrm{~B})$ at 3 or 5 years $(6 \%$ or $5 \%$, respectively). Also common was to begin in the nonatopic, asthma and rhinitis symptoms phenotype $1 \mathrm{C}$ at 1.5 and 3 years, and transition to the nonatopic, few symptoms/no respiratory disease phenotype (1A or $2 \mathrm{~A}$ ) from 3 or 5 years onwards ( $5 \%$ or $6 \%$ respectively).

\section{Predictors of phenotypes}

Table 4 shows the relationship between potential risk factors for respiratory disease and phenotypes at each age. There was no association between sex and phenotype except at 11.5 years, when boys were less likely than girls to belong to the atopic asthma phenotype (2D) (OR 0.52, 95\% CI 0.29-0.92). In general, the randomised interventions did not influence phenotype membership. Children of mothers who smoked during pregnancy were less likely to belong to the atopic, few symptoms phenotype (1B or $2 \mathrm{~B})$ at 5 years (OR 0.33 , 95\% CI $0.15-0.74$ ) and 11.5 years (OR 0.49, 95\% CI $0.27-0.90$ ) and more likely to belong to the nonatopic, asthma and rhinitis phenotype (1C) at 1.5 years (OR 1.91, 95\% CI 1.14-3.19). Children exposed to household tobacco smoke were also less likely to belong to the atopic, few symptoms phenotype (1B) at 5 years (OR 0.42, 95\% CI 0.18-0.99). Children who were breastfed for at least 6 months were more likely to belong to the atopic, few symptoms phenotype (1B) at 1.5 years (OR 5.14, 95\% CI 1.09-24.34), but this measure of breastfeeding duration did not influence phenotype at later ages. Children who had eczema at 1.5 years were generally more likely to belong to either one or both of the atopy phenotypes $(1 \mathrm{~B}, 1 \mathrm{D}, 2 \mathrm{~B}$ and $2 \mathrm{D})$ at all ages than to the nonatopic, few symptoms phenotype (1A or $2 \mathrm{~A}$ ). Those with older siblings were less likely to belong to atopic, few symptoms phenotype (1B) at 5 years (OR $0.47,95 \%$ CI $0.27-0.81$ ) and the atopic, asthma phenotype (2D) at 8 years (OR 0.51, 95\% CI 0.29-0.92), but more likely to belong to the nonatopic, asthma and rhinitis symptoms phenotype (1C) at 1.5 years(OR 2.18, 95\% CI 1.33-3.58). Children of tertiary educated parents were less likely to belong to the nonatopic, asthma and rhinitis symptoms phenotype (1C) at 1.5 years (mothers OR $0.49,95 \%$ CI $0.31-0.76 /$ fathers OR $0.57,95 \%$ CI 0.36-0.89), but this aspect of socioeconomic status had no other effects on phenotype.

\section{Discussion}

We have shown heterogeneity in the manifestations of asthma and related respiratory disease during early and mid-childhood that is represented by four phenotypes in both periods. These can be described as 1) nonatopic, few symptoms, 2) atopic, few symptoms, 3) nonatopic, asthma and rhinitis symptoms, and 4) atopic, asthma and rhinitis symptoms in early childhood; and 1) nonatopic, no respiratory disease, 2) atopic, no respiratory disease, 3) nonatopic, asthma symptoms, no AHR or airway inflammation and 4) atopic asthma in mid-childhood. Transitioning between phenotypes was common in early childhood, but less common in later childhood. While maternal smoking during pregnancy and duration of 
breastfeeding influenced phenotypic class membership in early childhood, these and other risk factors had little influence in mid-childhood.

Our study extends previous attempts to define respiratory disease phenotypes in childhood in two ways: 1) by extending longitudinal analyses of a single respiratory symptom (wheeze) [2-4] to include repeated measures of several manifestations of respiratory disease and define phenotypes at multiple time points, and 2) by extending cross-sectional studies of multiple manifestations of respiratory disease [8-12] to a longitudinal analysis that includes estimation of transition probabilities. To the best of our knowledge, this study is the first to simultaneously define phenotypes with multiple disease manifestations at more than two time points and examine transitions between phenotypes over time in a population-based sample of children using a data-driven approach. This approach addresses one of the basic challenges of phenotype definition: whether change of features over time should be regarded as intrinsic to a particular phenotype (as shown in earlier studies by HENDERSON et al. [4] and SpYCHER et al. [6]) or as evidence of different phenotypes at different ages (as shown in cross-sectional studies by JUsT et al. [18]), i.e. transition between phenotypes. The LTA model is a novel method for addressing the question of how manifestations of respiratory disease in childhood change over time by reducing a large number of repeated observations in a cohort into meaningful patterns. Furthermore, it differs from previous longitudinal analyses of phenotypes that include multiple disease manifestations $[6,7]$ in explicitly modelling transitions rather than trajectories. This avoids the assumption that all members of a phenotype transition in the same way over time. Our figure 1 highlights the value of this approach. A further strength is the detailed clinical assessment of this cohort using questionnaire data and objective measures of atopy, lung function and airway inflammation at five ages between 1.5 and 11.5 years.

The phenotypes that we have described in children aged 1.5-11.5 years can be compared with phenotypes in previous cross-sectional studies in children. In terms of the optimal number of phenotypes to describe the heterogeneity, our study found four phenotypes. This is within the range observed in other studies (between three and seven phenotypes) $[6,8-12,18]$. In early childhood, three of the four phenotypes in our study are similar to those (asymptomatic, atopic severe and nonatopic severe) described in a cross-sectional study of children aged 18 months which used similar attributes to ours in their model [8]. However, they did not find an asymptomatic atopy phenotype (1B) as we did. This may be due to the absence of children older than 18 months. Other studies in preschool children cannot be compared directly with ours because they were limited to children with asthma or had measured different attributes to those used in our study $[11,18]$. Only one other study conducted in the mid-childhood age range has examined asthma phenotypes in a general population sample [10]. Nearly all other studies have been restricted to populations of children with asthma $[9,12,33,34]$. In common with our study, others have found that respiratory symptoms [10], atopy $[9,12,33,34]$, AHR $[9,12]$ and airway inflammation $[9,34]$ are distinguishing features of phenotypes. Hence, the findings of this study conducted during early and mid-childhood are consistent with previous cross-sectional studies conducted within this age range.

One of the most interesting findings of this analysis is the existence and trajectory of the phenotype labelled as "nonatopic, asthma and rhinitis symptoms" in early childhood and "nonatopic, asthma symptoms, no AHR or airway inflammation" in mid-childhood. This represents over one-third of all cohort members at age 18 months, but only $8 \%$ of cohort members at age 11.5 years. Most of those with this phenotype in early childhood transition to the "nonatopic, few symptoms" phenotype; in other words, they remit. This time course is reminiscent of the transient early wheeze first described by MARTINEZ et al. [1], and subsequently identified by Henderson et al. [4] and SAVEnije et al. [2] in the ALSPAC (Avon Longitudinal Study of Parents And Children) and PIAMA (Prevention and Incidence of Asthma and Mite Allergy) cohorts. This phenotype also probably corresponds to the "Episodic viral wheeze" group identified in a recent review of phenotypes by JUst et al. [35] who, like our class "C", have a good prognosis and low airway inflammation. Our analysis adds weight to the view that this common early-life wheezing phenotype has a benign prognosis. The absence of atopy is the best existing marker for membership of this phenotype, but better early-life markers are needed.

BOUDIER et al. [20] conducted a similar analysis to that presented here using combined data from three large adult cohorts, studied over a 10-year interval. They were able to identify more subgroups than the present study. The major difference in methodology from the present analysis, apart from the facts that it was conducted in adults rather than children and that the study population was much larger, is that it was limited to subjects who were identified as having asthma based on predetermined criteria. This limitation may have facilitated identification of more subgroups within the population with asthma. Since we did not wish to pre-determine what constituted "asthma", our analysis included an entire community-based cohort, including children with and without asthma, and allowed the model to define "asthma" phenotypes within this cohort. A further strength of the present study was that we were able observe cohort members on five occasions over a 10-year period, in contrast to just two occasions in the adult cohort. 
It should be noted that the labels that we have attached to the data-derived classes (phenotypes), although based on examination of the item-response probabilities, are essentially arbitrary. This is a common feature of all phenotypes that are derived using unsupervised, data-driven statistical approaches [6, 8-12]. Readers can use the information provided in table 3 to make their own decision about the most appropriate labels for each of the phenotypes we have identified.

The trajectories and transitions that we have described for multicomponent phenotypes derived by a data-driven approach, i.e. LTA, can be compared with previously described studies of prognosis which used arbitrarily defined phenotypes. The Melbourne Asthma Study explored transitions in a cohort of children with asthma recruited at age 7 years followed to adulthood [36, 37]. Our results from 3 to 11.5 years are consistent with their finding, over a longer follow-up period, that those in the least severe or most severe disease groups had the highest chance of remaining in these groups over time, compared with subjects in other more infrequent/intermediate disease groups, who tended to transition between groups over time.

Our study can also be compared with previous studies on the natural history of wheezing illness in early to mid-childhood. A study in preschool children, conducted over a 12-month period, found classification of children as having episodic viral wheeze or multiple trigger wheeze was not stable over this short period [17]. In contrast, another study in preschool children found that, although remission was common if a child was classified as having mild disease, no remission was observed in a more severe atopic phenotype and those with nonatopic uncontrolled wheeze commonly transitioned to atopic uncontrolled wheeze [18]. However, in contrast to our study, they did not examine transition probabilities within a single longitudinal model, but instead fitted separate models at each age. Furthermore, they examined children over a relatively short, 1- or 2-year age range, in contrast to our 10 years. Hence, although our results are consistent with previous studies, they represent a substantial advance on previous analyses.

The transitions of children through phenotypes and changes in phenotype membership prevalence highlight the importance of age when defining childhood asthma phenotypes. The unstable nature of phenotypes at 1.5 and 3 years of age could be due to a preschool effect, in which children are first exposed to infections and develop respiratory symptoms [38]. The increase in prevalence of the atopic phenotypes $(1 \mathrm{~B}, 2 \mathrm{~B}, 1 \mathrm{D}$ and $2 \mathrm{D})$ reflects the acquisition of allergic sensitisation with age in children [39]. Our findings are consistent with the previous observation that atopy is associated with more persistent and severe respiratory disease in childhood [16], and that this persistent disease could begin as young as age 18 months. The finding that $30 \%$ of our sample who were classified as "atopic, asthma and rhinitis symptoms" phenotype at 1.5 years did not belong to the similar atopic asthma phenotype at 11.5 years also supports the common observation that some children grow out of wheezing symptoms in early life, while others progress to develop asthma [40].

We tested several potential risk factors for class membership. We found children were less likely to belong to the "nonatopic, asthma and rhinitis" phenotype at 1.5 years if they were breastfed for $\geqslant 6$ months or had better educated parents and were more likely to belong to this phenotype if they had older siblings or mothers who smoked in pregnancy. These findings are consistent with previous reports on risk factors for early life wheezing illness $[38,41,42]$. Our results support studies that have found that eczema in early life is a risk factor for asthma in later childhood [43]. Prior to 11.5 years, phenotype membership did not differ between boys and girls. However, at 11.5 years, boys were less likely than girls to belong to the "atopic asthma" phenotype. These findings are in contrast to previous studies, which found that wheeze and asthma are more common in boys than girls before puberty [44]. It is possible that the selected nature of this cohort, in which all subjects had a family history of asthma, masked the sex differences that are apparent in general population cohorts.

Although the sample size for this analysis was relatively small, the use of data from five repeated measures adds substantially to the ability of the LTA to define phenotypes and their transitions [29, 45]. Furthermore, the time between the assessments was relatively short (1.5-3.5 years), allowing the model to incorporate the variability in respiratory disease manifestations over time. We acknowledge that the phenotype definitions are limited by the variables that were included in the analysis. Thus, it is possible other patterns might have emerged if other variables were included. However, the four phenotypes, as defined, are clinically relevant and consistent with the observation there is a spectrum of asthma in childhood $[46,47]$. Nevertheless, the sample size is small and the study is exploratory, and hence requires external validation in other cohorts. Further discussion of sample size issues is included in the online supplementary material.

The CAPS cohort is a high-risk cohort for asthma by virtue of the family history of asthma. Hence, the prevalence of phenotypes observed in this population may not be generalisable to other populations. However, the use of this population may have increased our capacity to identify uncommon phenotypes in the general population. Furthermore this was not a highly selected cohort as nearly one-third of those 
screened met the eligibility criteria [23] and at 8 years the prevalence of asthma in this cohort is only slightly higher than in the general population [24]. $40 \%$ of the initially recruited cohort did not participate at the 11.5-year assessment. We have previously shown that mothers of respondents at 11.5 years were less likely to smoke during pregnancy, and both mothers and fathers were older, more highly educated and more likely to be employed full-time than parents of nonrespondents [25]. Hence, there is a risk of selection bias in this cohort. This may have had some effect of the definition and prevalence of phenotypes.

In conclusion, we have provided quantitative support for the common observation that asthma and related respiratory disease in children is a heterogeneous entity, and some children with wheeze and other respiratory symptoms in early life progress to asthma in mid-childhood, while others become asymptomatic. This approach will be useful for defining phenotypic end points in future studies examining environmental and genetic risk factors for chronic and recurrent respiratory disease in children. Clinically, however, we will continue to have difficulty predicting in individual children, especially in early infancy, their likelihood of persistence of wheeze and asthma.

\section{Acknowledgements}

We would like to thank the reviewers for their thoughtful comments during the review process which helped us to improve our paper.

\section{References}

1 Martinez FD, Wright AL, Taussig LM, et al. Asthma and wheezing in the first six years of life. N Engl J Med 1995; 332: 133-138.

2 Savenije OE, Granell R, Caudri D, et al. Comparison of childhood wheezing phenotypes in 2 birth cohorts: ALSPAC and PIAMA. J Allergy Clin Immunol 2011; 127: 1505-1512.

3 Lodge CJ, Zaloumis S, Lowe AJ, et al. Early-life risk factors for childhood wheeze phenotypes in a high-risk birth cohort. J Pediatr 2014; 164: 289-294.

4 Henderson J, Granell R, Heron J, et al. Associations of wheezing phenotypes in the first 6 years of life with atopy, lung function and airway responsiveness in mid-childhood. Thorax 2008; 63: 974-980.

5 Rancière F, Nikasinovic L, Bousquet J, et al. Onset and persistence of respiratory/allergic symptoms in preschoolers: new insights from the PARIS birth cohort. Allergy 2013; 68: 1158-1167.

6 Spycher BD, Silverman M, Pescatore AM, et al. Comparison of phenotypes of childhood wheeze and cough in 2 independent cohorts. J Allergy Clin Immunol 2013; 132: 1058-1067.

7 Belgrave DCM, Granell R, Simpson A, et al. Developmental profiles of eczema, wheeze, and rhinitis: two population-based birth cohort studies. PLoS Med 2014; 11: e1001748.

8 Herr M, Just J, Nikasinovic L, et al. Risk factors and characteristics of respiratory and allergic phenotypes in early childhood. J Allergy Clin Immunol 2012; 130: 389-396.

9 Fitzpatrick AM, Teague WG, Meyers DA, et al. Heterogeneity of severe asthma in childhood: confirmation by cluster analysis of children in the National Institutes of Health/National Heart, Lung, and Blood Institute Severe Asthma Research Program. J Allergy Clin Immunol 2011; 127: 382-389.

10 Weinmayr G, Keller F, Kleiner A, et al. Asthma phenotypes identified by latent class analysis in the ISAAC phase II Spain study. Clin Exp Allergy 2013; 43: 223-232.

11 Just J, Gouvis-Echraghi R, Couderc R, et al. Novel severe wheezy young children phenotypes: boys atopic multiple-trigger and girls nonatopic uncontrolled wheeze. J Allergy Clin Immunol 2012; 130: 103-110.

12 Howrylak JA, Fuhlbrigge AL, Strunk RC, et al. Classification of childhood asthma phenotypes and long-term clinical responses to inhaled anti-inflammatory medications. J Allergy Clin Immunol 2014; 133: 1289-1300.

13 Henderson J, Granell R, Heron J, et al. Associations of wheezing phenotypes in the first 6 years of life with atopy, lung function and airway responsiveness in mid-childhood. Thorax 2008; 63: 974-980.

14 Brand PLP, Baraldi E, Bisgaard H, et al. Definition, assessment and treatment of wheezing disorders in preschool children: an evidence-based approach. Eur Respir J 2008; 32: 1096-1110.

15 Depner M, Fuchs O, Genuneit J, et al. Clinical and epidemiologic phenotypes of childhood asthma. Am J Respir Crit Care Med 2013; 189: 129-138.

16 Xuan W, Marks GB, Toelle BG, et al. Risk factors for onset and remission of atopy, wheeze, and airway hyperresponsiveness. Thorax 2002; 57: 104-109.

17 Schultz A, Devadason SG, Savenije OE, et al. The transient value of classifying preschool wheeze into episodic viral wheeze and multiple trigger wheeze. Acta Paediatrica 2010; 99: 56-60.

18 Just J, Saint-Pierre P, Gouvis-Echraghi R, et al. Wheeze phenotypes in young children have different courses during the preschool period. Ann Allergy Asthma Immunol 2013; 111: 256-261.

19 Soto-Ramirez N, Ziyab AH, Karmaus W, et al. Epidemiologic methods of assessing asthma and wheezing episodes in longitudinal studies: measures of change and stability. J Epidemiol 2013; 23: 399-410.

20 Boudier A, Curjuric I, Basagana X, et al. Ten-year follow-up of cluster-based asthma phenotypes in adults. a pooled analysis of three cohorts. Am J Respir Crit Care Med 2013; 188: 550-560.

21 Marks GB, Mihrshahi S, Kemp AS, et al. Prevention of asthma during the first 5 years of life: a randomized controlled trial. J Allergy Clin Immunol 2006; 118: 53-61.

22 Mihrshahi S, Peat JK, Webb K, et al. The Childhood Asthma Prevention Study (CAPS): design and research protocol of a randomized trial for the primary prevention of asthma. Control Clin Trials 2001; 22: 333-354.

23 Mihrshahi S, Vukasin N, Forbes S, et al. Are you busy for the next 5 years? Recruitment in the Childhood Asthma Prevention Study (CAPS). Respirology 2002; 7: 147-151.

24 Toelle BG, Ng KKW, Crisafulli D, et al. Eight-year outcomes of the Childhood Asthma Prevention Study. J Allergy Clin Immunol 2010; 126: 388-389.

25 Toelle BG, Garden FL, Ng KKW, et al. Outcomes of the Childhood Asthma Prevention Study at 11.5 years. J Allergy Clin Immunol 2013; 132: 1220-1222. 

separate steps in the 'allergic march'. Clin Exp Allergy 2007; 37: 1296-1302.

27 Lowe AJ, Abramson MJ, Hosking CS, et al. The temporal sequence of allergic sensitization and onset of infantile eczema. Clin Exp Allergy 2007; 37: 536-542.

28 Schuttelaar MLA, Kerkhof M, Jonkman MF, et al. Filaggrin mutations in the onset of eczema, sensitization, asthma, hay fever and the interaction with cat exposure. Allergy 2009; 64: 1758-1765.

29 Collins LM, Lanza ST. Latent class and latent transition analysis: with applications in the social, behavioral, and health sciences. Hoboken, Wiley, 2010.

30 Muthen LK, Muthen BO. Mplus user's guide. 7th Edn. Los Angeles, Muthen \& Muthen, 2012.

31 Cumsille P, Darling N, Flaherty B, et al. Heterogeneity and change in the patterning of adolescents' perceptions of the legitimacy of parental authority: a latent transition model. Child Dev 2009; 80: 418-432.

32 Celeux G, Soromenho G. An entropy criterion for assessing the number of clusters in a mixture model. J Classif 1996; 13: 195-212.

33 Schatz M, Hsu JW, Zeiger RS, et al. Phenotypes determined by cluster analysis in severe or difficult-to-treat asthma. J Allergy Clin Immunol 2014; 133: 1549-1556.

34 Just J, Saint-Pierre P, Gouvis-Echraghi R, et al. Childhood allergic asthma is not a single phenotype. J Pediatr 2014; 164: 815-820.

35 Just J, Saint Pierre P, Amat F, et al. What lessons can be learned about asthma phenotypes in children from cohort studies? Pediatr Allergy Immunol 2015; 26: 300-305.

36 Wolfe R, Carlin JB, Oswald H, et al. Association between allergy and asthma from childhood to middle adulthood in an Australian cohort study. Am J Respir Crit Care Med 2000; 162: 2177-2181.

37 Phelan PD, Robertson CF, Olinsky A. The Melbourne asthma study: 1964-1999. J Allergy Clin Immunol 2002; 109: 189-194.

38 Ball TM, Castro-Rodriguez JA, Griffith KA, et al. Siblings, day-care attendance, and the risk of asthma and wheezing during childhood. N Engl J Med 2000; 343: 538-543.

39 Kulig M, Bergmann R, Klettke U, et al. Natural course of sensitization to food and inhalant allergens during the first 6 years of life. J Allergy Clin Immunol 1999; 103: 1173-1179.

40 Sears MR, Greene JM, Willan AR, et al. A longitudinal, population-based, cohort study of childhood asthma followed to adulthood. N Engl J Med 2003; 349: 1414-1422.

41 Dell S, To T. Breastfeeding and asthma in young children: findings from a population-based study. Arch Pediatr Adolesc Med 2001; 155: 1261-1265.

42 Dom S, Droste JHJ, Sariachvili MA, et al. The influence of parental educational level on the development of atopic sensitization, wheezing and eczema during the first year of life. Pediatr Allergy Immunol 2009; 20: 438-447.

43 Anderson HR, Pottier AC, Strachan DP. Asthma from birth to age 23: incidence and relation to prior and concurrent atopic disease. Thorax 1992; 47: 537-542.

44 Mandhane PJ. Sex differences in factors associated with childhood- and adolescent-onset wheeze. Am J Respir Crit Care Med 2005; 172: 45-54.

45 Wang J, Wang X. Structural equation modeling: applications using Mplus. Chichester, Wiley, 2012.

46 McNicol KN, Williams HB. Spectrum of asthma in children, clinical and physiological components. BMJ 1973; 4 : 7-11.

47 Martinez FD. Development of wheezing disorders and asthma in preschool children. Pediatrics 2002; 109: $362-367$. 